\title{
Biallelic mutations in $C D C 20$ cause female infertility characterized by abnormalities in oocyte maturation and early embryonic development
}

\section{Dear Editor,}

Previously, the Mendelian phenotypes in human oocyte maturation arrest, fertilization failure and early embryonic arrest, are largely underestimated. In recent years, "missing" Mendelian phenotypes and genes in these processes are beginning to be uncovered by us and others (Huang et al., 2014; Alazami et al., 2015; Feng et al., 2016; Xu et al., 2016; Chen et al., 2017; Sang et al., 2019). However, the genetic basis for majority of patients resulting from abnormalities in these phenotypes remains to be elucidated.

The cell division cycle 20 (CDC20, HGNC:1723) is the coactivator of anaphase-promoting complex/cyclosome (APC/ C) during mitosis, and plays a role in maintaining the genome by regulating spindle assembly checkpoints (Musacchio and Hardwick, 2002). In oocytes, the activation of APC/C by CDC20 is a key step in homologue disjunction and in transition from meiosis I to meiosis II (Jones, 2011). CDC20 is therefore an essential component of the mammalian cell cycle mechanism regulating both miotic and meiotic exit. Although $C D C 20$ is an extensively studied gene, until now, no solid evidence has been provided to establish the causal relationship between $C D C 20$ mutations and human diseases. The only report was an association study relating CDC20 mutations with idiopathic azoospermia ( $\mathrm{Li}$ et al., 2017).

Here, we identified biallelic CDC20 mutations in five infertile individuals with oocyte maturation arrest, fertilization failure, and early embryonic arrest. We investigated the effects of the corresponding mutations in cell lines and mouse oocytes and explored a potential therapeutic treatment by direct $C D C 20$ cRNA injection.

The probands in families 1 and 2 had normal menstrual cycles and had been diagnosed as primary infertility with unknown reasons for several years (Table S1). Both of the probands came from consanguineous families (Fig. 1A). The probands in family 1 had undergone two failed in vitro fertilization (IVF) attempts and one failed intracytoplasmic sperm injection (ICSI). In her ICSI cycle, eleven oocytes were obtained, and ten oocytes were arrested at the germinal vesicle (GV) or metaphase I (MI) stage (Fig. 1B), only one was matured and fertilized, but was arrested at threecell stage (Table S1). The proband in family 2 had undergone two failed ICSI cycles. All of the retrieved oocytes were arrested at the Ml stage (Table S1). In summary, both two probands had phenotypes of oocyte maturation arrest in their ICSI attempts. Whole-exome sequencing and homozygosity mapping was performed (Fig. S1). By a recessive inheritance model, we identified two homozygous missense mutations (c.683A $>\mathrm{G}$, p.Tyr228Cys and c.1316T>G, p.Leu439Arg) in CDC20, respectively (Fig. 1A). Because the parents of both probands were unavailable, in order to rule out the possibility that the homozygous status of CDC20 resulted from the deletion of one allele, we performed a copy number variation (CNV) analysis. There was no CNV for CDC20 in the probands of both families, which confirmed that the mutations in the two individuals were indeed homozygous (Fig. S2A and S2B).

Mutational screening of $C D C 20$ were pursed in a large cohort of 1,250 infertile individuals with abnormalities in oocyte maturation, fertilization and early embryonic development by whole exome sequencing. Additional biallelic mutations in $C D C 20$ were detected in another three individuals from families 3-5 (Fig. 1A). The compound heterozygous mutations in $C D C 20$ in the affected individual in family 3 consisted of a missense mutation c.965G >A ( $p$. Arg322GIn) and a nonsense mutation c.544C $>T$ (p. Arg182*). The proband in family 4 carried a compound heterozygous mutation consisting of a missense mutation c.965G >A (p.Arg322GIn) and a 4 bp insertion c.813_814ins AGTG (p.Gly272Serfs ${ }^{\star} 24$ ). The proband in family 5 carried a compound heterozygous mutation consisting of a missense mutation c.965G>A (p.Arg322GIn) and a 4 bp deletion c.1176_1179del TCTG (p.Cys392*). Sanger sequencing were performed to confirm the mutations in these three families (Fig. 1A). Information about the mutations is shown in Fig. $1 \mathrm{~A}$ and Table S2, and the positions of the mutations 


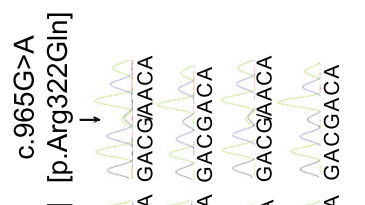



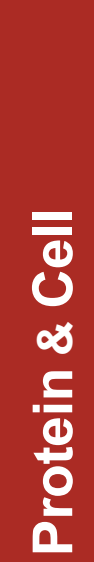


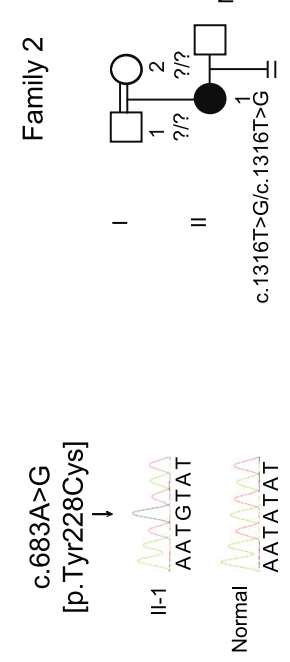

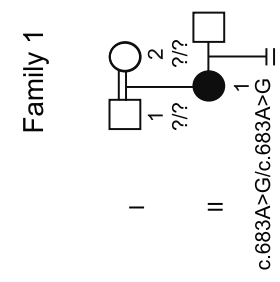

$\varangle$
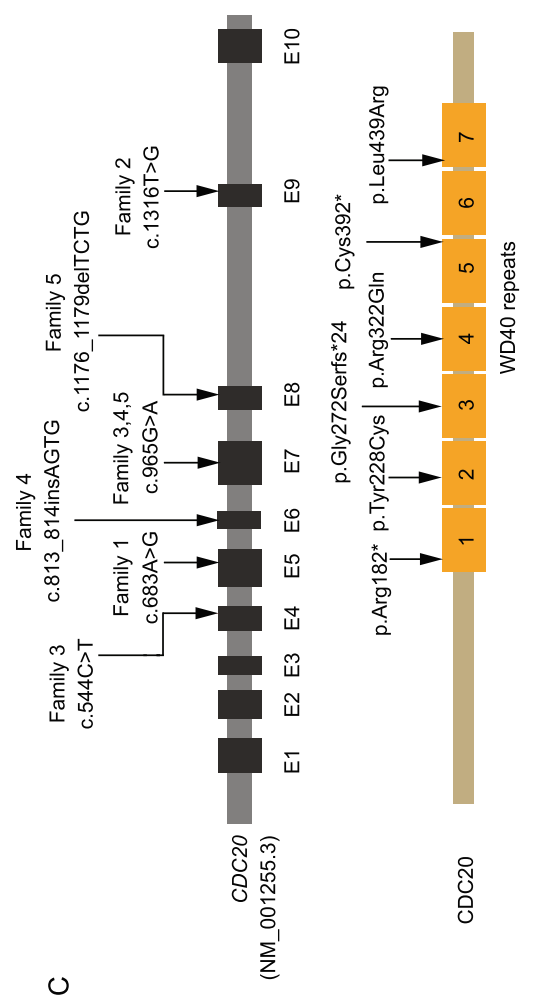

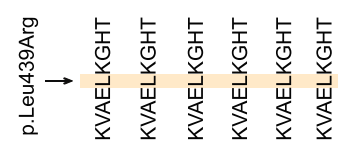

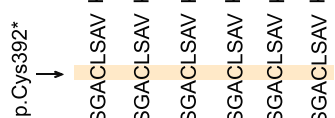

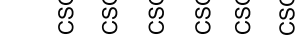

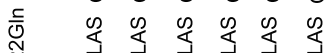

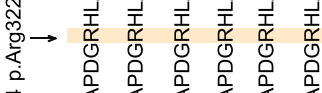

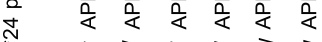

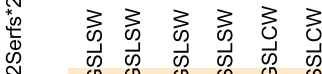



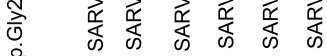

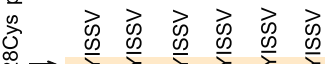

离 $\rightarrow$ 至

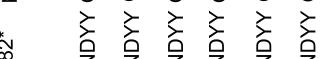

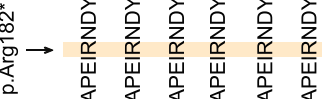

IIIs
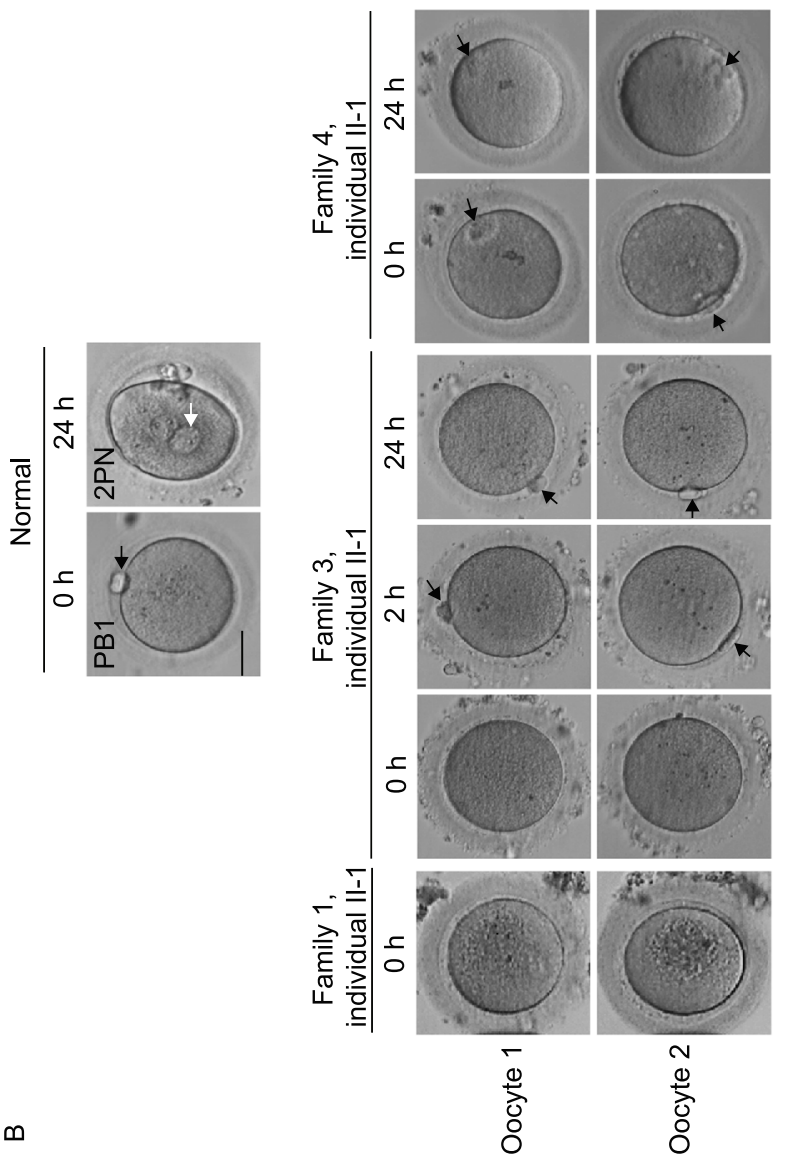
Figure 1. Identification of mutations in $C D C 20$ and effects of the mutations on protein level of CDC20 and cyclin B1. (A) Five pedigrees presented with abnormalities in oocyte maturation, fertilization and early embryonic development. Question marks indicate unavailable DNA samples, double lines denote consanguineous marriage, the equal sign denote infertility. (B) The morphology of normal and affected individual oocytes at $0 \mathrm{~h}, 2 \mathrm{~h}$ and $24 \mathrm{~h}$ after retrieved. The black arrows indicate the first polar body (PB1), and the white arrow in the normal oocyte indicates the pronuclei. Scale bar $=40 \mu \mathrm{m}$. (C) Locations and conservation of mutations in CDC20. (D) The effects of the mutations on $\mathrm{CDC} 20$ and cyclin B1 protein level by Western blot in transfected $\mathrm{CHO}$ cells. (E) Left: Quantitation of blank, wild type (WT) and mutant CDC20 protein level in Fig. 1D. The data are shown as means and SD. ${ }^{*} P<0.01$, ${ }^{* * \star} P<0.001$, ns, not significant. Right: Quantitation of cyclin B1 protein level of overexpression the WT and mutant CDC20 in Fig. 1D. The data are shown as means and SD. ${ }^{*} P<0.05$; ns, not significant. The experiment was performed with three independent biological replicates yielding similar results. (F) The effects of the mutations of p.Arg182* and p.Arg322GIn on CDC20 protein level by Western blot in LCLs of individual II-1 in family 3.

and their conservation in different species are shown in Fig. 1C. In family 3, oocytes were immature when retrieved. Most of immature oocytes could develop into the first polar body (PB1) oocytes after 2 hours' in vitro culture, but showed fertilization failure or early embryonic arrest. The proband in family 4 and family 5 had phenotypes of fertilization failure or early embryonic arrest. The specific clinical information is indicated in Fig. 1B and Table S1.

In transfected Chinese hamster ovary $(\mathrm{CHO})$ cells, the missense mutations p.Tyr228Cys and p.Leu439Arg resulted in a reduction in CDC20 protein level, while mutations $p$. Arg $182 *^{*}$, p.Gly272Serfs ${ }^{*} 24$, and p.Cys392* resulted in truncated proteins (Fig. 1D and 1E). For mutation p. Arg322GIn, though there was no obvious effect on CDC20 protein level in $\mathrm{CHO}$ cells, the Western blot analysis of lymphoblastoid cell line (LCL) of the affected individual II in family 3 with a missense mutation c.965G>A (p. Arg322GIn) and a nonsense mutation c.544C>T (p. Arg182*) showed significantly reduced protein level (Figs. $1 F$ and S3A). In addition, the mRNA expression of CDC20 in the LCLs of individual II-1 in family 3 and in the granulosa cells (GCs) of individual II-1 in family 4 were also reduced significantly (Fig. S3B and S3C). We also explored the effects of the mutations (c.683A>G, c. 813_814ins AGTG, c.1176_1179del TCTG, and c. 1316T>G) on mRNA expression in transfected $\mathrm{CHO}$ cells, and the results showed that all these four mutations caused significantly reduced mRNA expression (Fig. S4). All these results indicate that mutations in $C D C 20$ lead to unstable protein and degraded RNA per se. During the metaphase to anaphase transition, APC/C is activated through the release from $\mathrm{CDC} 20$ inhibition, and this leads to



丩

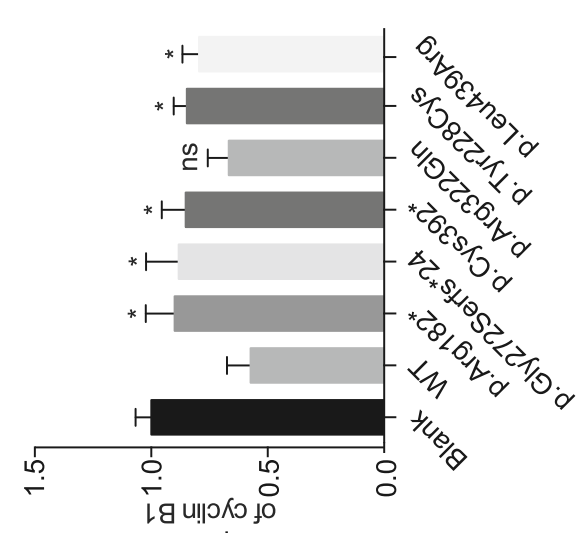

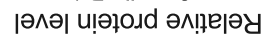

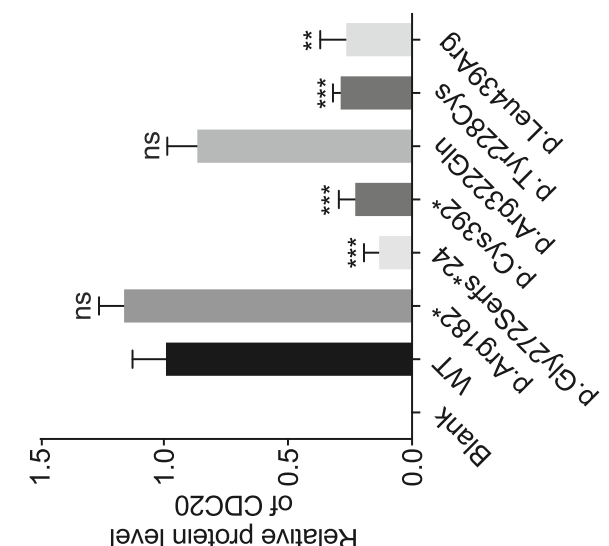

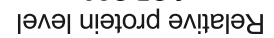
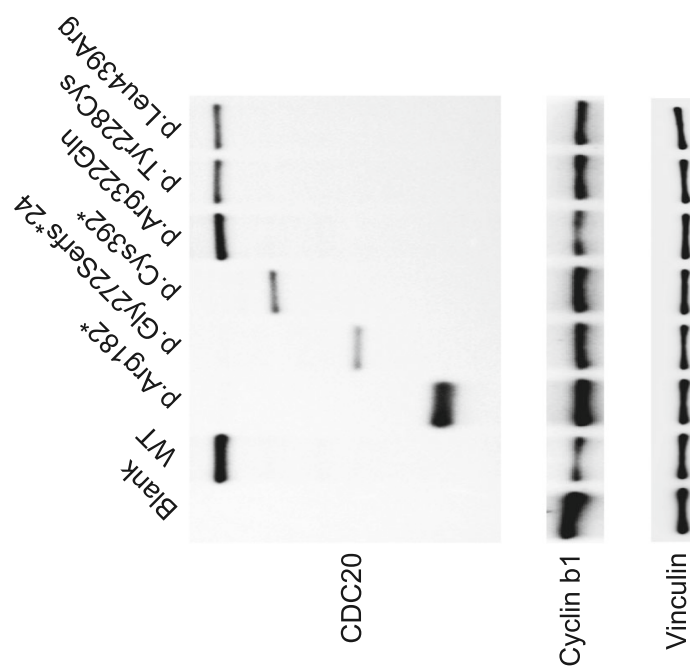

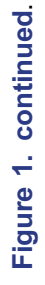



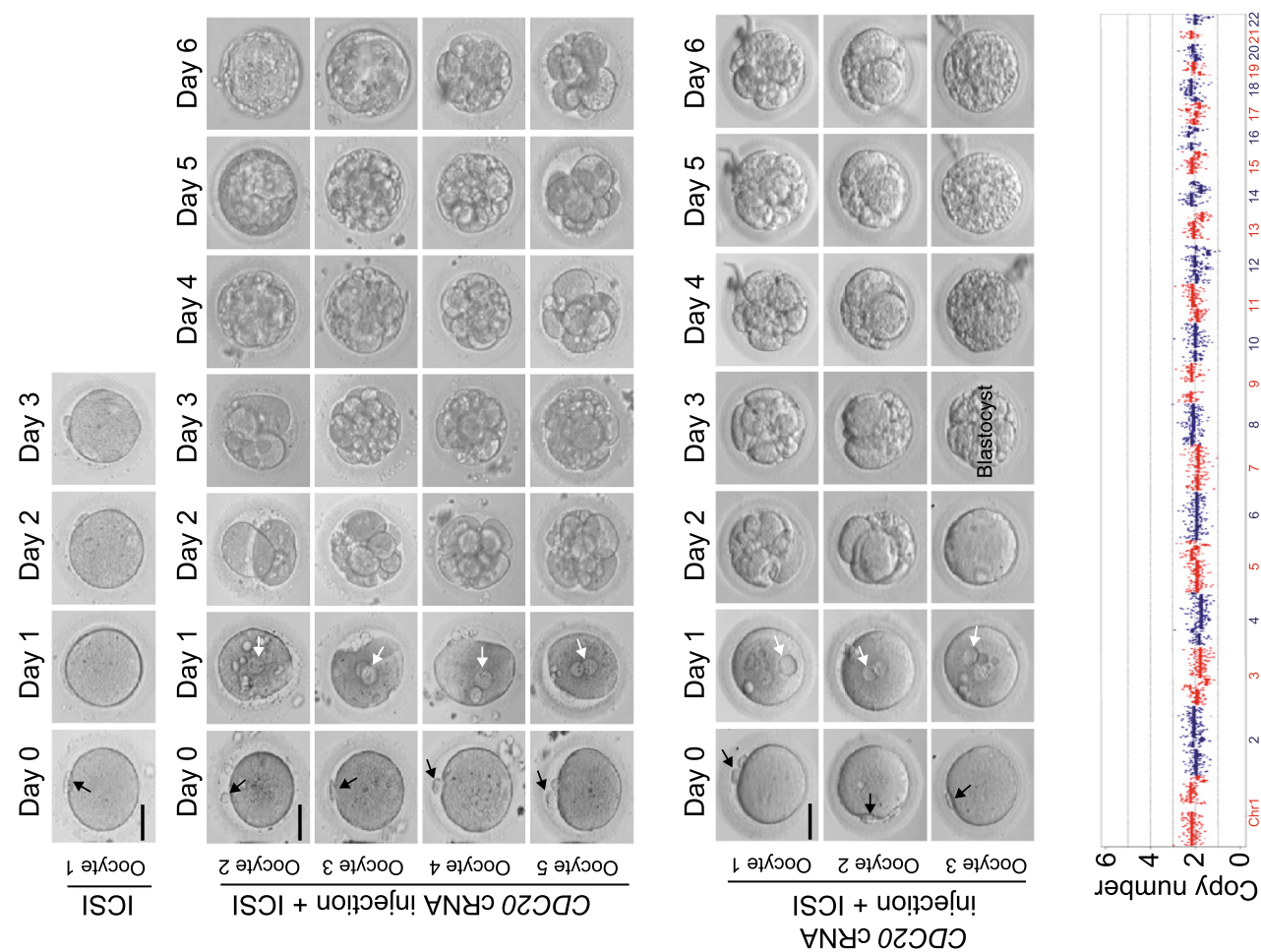

U
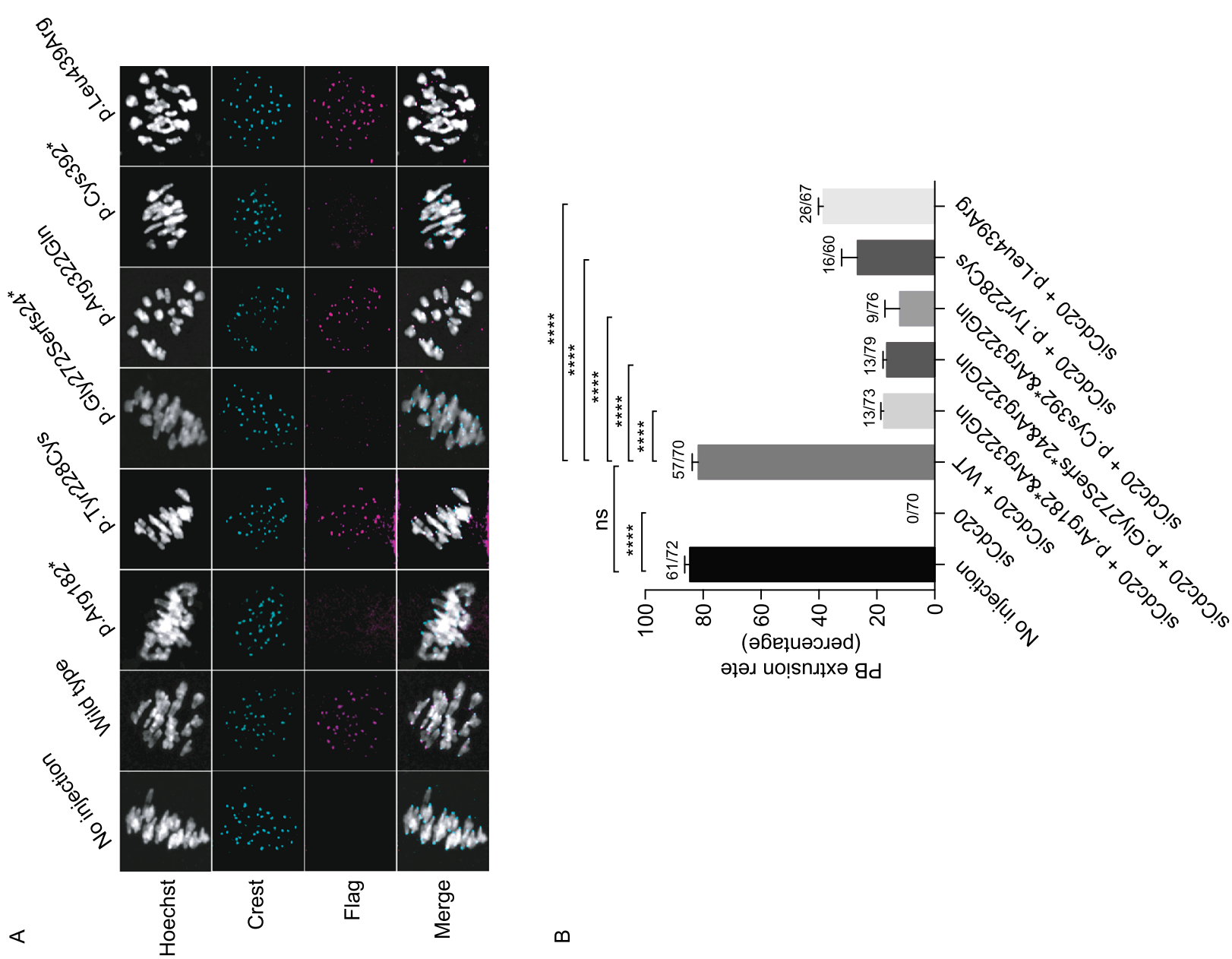
4 Figure 2. Effects of mutations on CDC20 localization and function in mouse oocytes and phenotypic rescue by CDC20 cRNA injection into oocytes from the proband of family 3 and family 4 . (A) Localization of WT and mutant FLAG-tagged CDC20 in mouse oocytes. Hoechst and Crest were used to label the DNA and kinetochores, respectively. (B) The effects of the mutations on the rescue of PB1 extrusion in $\mathrm{Cdc20}$ knockdown mouse oocytes with phenotype of $\mathrm{Ml}$ arrest. The number of oocytes used are listed on top of the column. Three independent experiments were performed. ${ }^{* * * *} P<0.0001$; ns, not significant. (C) One PB1 oocyte of individual II-1 in family 3 underwent ICSI without CDC20 cRNA injection was used as control. Day 0 indicates the time point at 4 $\mathrm{h}$ after ICSI. The black arrowhead indicates the polar body. Scale bar $=40 \mu \mathrm{m}$. (D) Four retrieved PB1 oocytes of individual II- 1 in family 3 were injected with CDC20 cRNA and cultured for $4 \mathrm{~h}$ and then used for ICSI. Oocytes were monitored for 6 days after ICSI. The white arrows indicate the pronuclei. (E)Three retrieved PB1 oocytes of individual II-1 in family 4 were injected with $C D C 20 \mathrm{cRNA}$ and cultured for $4 \mathrm{~h}$ and then used for ICSI. (F) Trophectoderm cells from the blastocyst embryo of individual II-I in family 3 were collected and sequencing for chromosomal copy number variation analysis.

the breakdown of cyclin B1 (HGNC:1579) (Nasmyth and Haering, 2005). We therefore determined the effects of $C D C 20$ mutations on cyclin B1 degradation. As shown in Fig. 1D and 1E, overexpression of wild-type CDC20 significantly decreased the endogenous protein level of cyclin B1, while most of mutations affected the degradation of cyclin B1.

We next investigated the effects of the mutations on human CDC20 localization in mouse oocytes. For the three missense mutations (p.Tyr228Cys, p.Arg322GIn, p. Leu439Arg), CDC20 showed normal kinetochore localization as the wild-type. In contrast, CDC20 failed to localize to the kinetochore for the other three nonsense or frameshift mutations (p.Arg182*, p.Gly272Serfs ${ }^{\star} 24, \quad$ p.Cys392*) (Fig. 2A), indicating these are loss-of-function mutations.

Knockdown of the Cdc20 gene exclusively blocks PB1 extrusion in mouse oocytes and the PB1 extrusion can be rescued by injecting Cdc20 cRNA into the oocytes (Reis et al., 2007). To further explore the effect of mutations on CDC20 function in oocytes, we first knocked down the endogenous $\mathrm{Cdc2O}$ in mouse oocytes by using $C d c 20$ siRNA, and we observed an $\mathrm{Ml}$ arrest phenotype that could be rescued by supplementation with human wild-type CDC20 cRNA (Fig. 2B). We then performed a rescue experiment using patient-derived mutant cRNAs in the same way. Compared with wild-type, all mutations significantly reduced the ability of $\mathrm{CDC} 20$ to rescue PB1 extrusion (Fig. 2B). These results indicate the overall impaired effect on $\mathrm{CDC} 20$ function of homozygous and compound heterozygous mutations.

We then explored a potential therapeutic treatment for two patients by $C D C 20$ cRNA injection. For the proband in family
3, compared with control, all four oocytes injected with CDC20 cRNA were successfully fertilized as indicated by the formation of two pronuclei on day 1 , and two of the oocytes developed into blastocysts on day 6 (Fig. $2 \mathrm{C}$ and 2D). For the proband in family 4 , all three oocytes injected with CDC20 cRNA were successfully fertilized on day 1 , and one developed into an eight-cell stage embryo (Fig. 2E). Preimplantation genetic screening showed that one of blastocysts in family 3 had normal numbers of chromosomes and no obvious large repetition/deletion fragments (Fig. 2F). These results provide a potential treatment for these patients in the future.

We found phenotypic variability among the affected individuals with $C D C 20$ mutations. In brief, the proband in family 1 and 2 had the phenotype of oocyte maturation arrest. Although the proband in family 3 had a slight delay in oocyte maturation, the ultimate phenotype in family 3 and 4 was characterized by fertilization failure. The proband in family 5 showed early embryonic arrest. It has been reported in mice that $\mathrm{Cdc} 20$ has the highest expression in metaphase II (MII) oocytes compared to GV and MI oocytes as well as early embryos (Amanai et al., 2006), and this expression pattern was also observed in our qRT-PCR results in human oocytes and early embryos (Fig. S5). It is therefore likely that different amounts of $\mathrm{CDC} 20$ are needed at different stages of oocyte maturation, fertilization, and early embryo development. As for the mutations we identified, both family 1 and 2 had homozygous missense mutations, while families 3-5 harbored heterozygous compound mutations including one missense mutation and either a nonsense mutation or a frameshift mutation. The combinations of various types of mutations may result in different degrees of impairment of CDC20 and thus could lead to phenotypic variability.

Although CDC20 plays an important role in both mitosis and meiosis (Musacchio and Hardwick, 2002; Jones, 2011), all affected individuals in the study only exhibited the phenotype of female infertility without any abnormalities in somatic tissues or organs. This might due to the different thresholds of CDC20 amount required between mitosis and meiosis (Fig. S5). In addition, previous studies showed that Cdc20 knockout mice were embryonic lethal, while Cdc20 hypomorphic mice with graded reduction of $\mathrm{CDC} 20$ protein level from $60 \%-27 \%$ were healthy and had a normal lifespan compared to wild type mice, but only female hypomorphic mice with $27 \%$ CDC20 protein level were infertile or subfertile (Jin et al., 2010; Malureanu et al., 2010). In contrast, in mitosis even profound reduction of CDC20 levels to $10 \%$ of normal still supports the onset of anaphase and the completion of mitosis (Wolthuis et al., 2008). All of these results indicate that female infertility resulting from $\mathrm{CDC} 20$ reduction is dosage dependent and that mitosis is more tolerant than meiosis to CDC20 reduction.

In summary, we identified biallelic mutations in $C D C 20$ responsible for variable phenotypes of female infertility characterized by abnormalities in oocyte maturation, fertilization and early embryonic development and implicated 
cRNA injection strategy for a potential therapeutic treatment for these patients.

\section{FOONOTES}

This work was supported by the National Key Research and Development Program of China (2016YFC1000600, 2017YFC10 01500 and 2018YFC1003800), the National Natural Science Foundation of China (Grant Nos. 81725006, 81822019, 81771581, 81971450 and 81971382 ), the project supported by Shanghai Municipal Science and Technology Major Project (2017SHZDZX01), Project of Shanghai Municipal Science and Technology Commission (19JC1411001), the Shanghai Rising Star Program (17QA1400200), the Natural Science Foundation of Shanghai (19ZR1444500, 17ZR1401900), Shuguang Program of Shanghai Education Development Foundation and Shanghai Municipal Education Commission (18SG03). The Strategic Collaborative Research Program of the Ferring Institute of Reproductive Medicine, Ferring Pharmaceuticals and Chinese Academy of Sciences (FIRMC200507). The capacity Building Planning Program for Shanghai Women and Children's Health Service. The collaborative innovation center project construction for Shanghai women and children's health.

Lei $W, Q S$ and $L Z$ conceived and designed the research study. Y K, S X, Ling W, X S, Z Y, J S, L S, Y X, Z Y, B L, X M, J F contributed to the recruitment, characterization, and oocyte imaging of the patients, $S X$ and $B$ L performed the mRNA injection of patients' oocytes. Q S performed the microinjection in mouse oocytes, $B C$ and $Q \mathrm{~L}$ contributed to the bioinformatics analysis. $\mathrm{L} Z$ performed the experiments, Jing $D$ provided the mouse keeping room, $S L$ and $X L$ contributed to the establishment of LCLs. J M, Wenjing W organized the medical records. $Z Z$, Jie $D$ and Weijie $W$ analysed the data. $L Z$, Lei $W$ and $Q S$ wrote the draft of this manuscript. $L J$ and $L H$ helped in editing and improving the manuscript. We thank the patients and their families for participating in this study, as well as the doctors for contributing to patients' samples collection and clinical support.

Lin Zhao, Songguo Xue, Zhongyuan Yao, Juanzi Shi, Biaobang Chen, Ling Wu, Lihua Sun, Yao Xu, Zheng Yan, Bin Li, Xiaoyan Mao, Jing Fu, Zhihua Zhang, Jian Mu, Wenjing Wang, Jing Du, Shuai Liu, Jie Dong, Weijie Wang, Qiaoli Li, Lin He, Li Jin, Xiaozhen Liang, Yanping Kuang, Xiaoxi Sun, Qing Sang, and Lei Wang declare that they have no conflict of interest.

All procedures followed were in accordance with the ethical standards of the ethical committee of the Shanghai East Hospital affiliated with Tongji University, the Ninth Hospital affiliated with Shanghai Jiao Tong University, IRB of the Medical College of Fudan University and with the Helsinki Declaration of 1975 , as revised in 2000 (5). Informed consent was obtained from all patients for being included in the study. All institutional and national guidelines for the care and use of laboratory animals were followed.

Lin Zhao ${ }^{1}$, Songguo Xue ${ }^{2}$, Zhongyuan $\mathrm{Yao}^{3}$, Juanzi Shi ${ }^{4}$, Biaobang Chen ${ }^{5}$, Ling $\mathrm{Wu}^{6}$, Lihua Sun ${ }^{2}$, Yao $\mathrm{Xu}^{2}$, Zheng $\mathrm{Yan}^{6}$, Bin $\mathrm{Li}^{6}$, Xiaoyan $\mathrm{Mao}^{6}$, Jing $\mathrm{Fu}^{7}$, Zhihua Zhang ${ }^{1}$, Jian $\mathrm{Mu}^{1}$, Wenjing Wang ${ }^{1}$, Jing $\mathrm{Du}^{5}$, Shuai Liu ${ }^{8}$, Jie Dong ${ }^{1}$, Weijie Wang ${ }^{1}$, Qiaoli $\mathrm{Li}^{1}$, Lin $\mathrm{He}^{9}$, Li $\operatorname{Jin}^{10}$, Xiaozhen Liang ${ }^{8}$, Yanping Kuang ${ }^{6}$, Xiaoxi Sun ${ }^{7}$, Lei Wang ${ }^{1,11,12 \bowtie}$ (D) Qing Sang ${ }^{1,11 \bowtie}$ (D)
${ }^{1}$ Institute of Pediatrics, Children's Hospital of Fudan University and the Shanghai Key Laboratory of Medical Epigenetics, the International Co-laboratory of Medical Epigenetics and metabolism, Ministry of Science and technology and Institutes of Biomedical Sciences, State Key Laboratory of Genetic Engineering, Fudan University, Shanghai 200032, China

2 Center of Assisted Reproduction, Shanghai East hospital, Tongji University, Shanghai 200120, China

3 The Reproductive Medical Center of Xiangya Hospital, Central South University, Changsha 41008, China

${ }^{4}$ Reproductive Medicine Center, Shaanxi Maternal and Child Care Service Center, Xi'an 710069, China

${ }^{5} \mathrm{NHC}$ Key Lab of Reproduction Regulation (Shanghai Institute of Planned Parenthood Research), Fudan University, Shanghai 200032, China

${ }^{6}$ Reproductive Medicine Center, Shanghai Ninth Hospital, Shanghai Jiao Tong University, Shanghai 200011, China

7 Shanghai Ji Ai Genetics and IVF Institute, Obstetrics and Gynecology Hospital, Fudan University, Shanghai 200011, China

${ }^{8}$ Key Laboratory of Molecular Virology Immunology, Institute Pasteur of Shanghai, Chinese Academy of Sciences, Shanghai 200031, China

${ }^{9}$ Bio-X Center, Key Laboratory for the Genetics of Developmental and Neuropsychiatric Disorders, Ministry of Education, Shanghai Jiao Tong University, Shanghai 200030, China

${ }^{10}$ State Key Laboratory of Genetic Engineering and Collaborative Innovation Center for Genetics and Development, School of Life Sciences, Fudan University, Shanghai 200438, China

11 Zhuhai Fudan Innovation Institute, Zhuhai 519000, China

12 Shanghai Center for Women and Children's Health, Shanghai 200062, China

$\triangle$ Correspondence: wangleiwanglei@fudan.edu.cn (L. Wang), sangqing@fudan.edu.cn (Q. Sang)

\section{OPEN ACCESS}

This article is licensed under a Creative Commons Attribution 4.0 International License, which permits use, sharing, adaptation, distribution and reproduction in any medium or format, as long as you give appropriate credit to the original author(s) and the source, provide a link to the Creative Commons licence, and indicate if changes were made. The images or other third party material in this article are included in the article's Creative Commons licence, unless indicated otherwise in a credit line to the material. If material is not included in the article's Creative Commons licence and your intended use is not permitted by statutory regulation or exceeds the permitted use, you will need to obtain permission directly from the copyright holder. To view a copy of this licence, visit http:// creativecommons.org/licenses/by/4.0/.

\section{REFERENCES}

Alazami AM, Awad SM, Coskun S, Al-Hassan S, Hijazi H, Abdulwahab FM, Poizat C, Alkuraya FS (2015) TLE6 mutation causes 
the earliest known human embryonic lethality. Genome Biol 16:240

Amanai M, Shoji S, Yoshida N, Brahmajosyula M, Perry AC (2006) Injection of mammalian metaphase II oocytes with short interfering RNAs to dissect meiotic and early mitotic events. Biol Reprod 75:891-898

Chen T, Bian Y, Liu X, Zhao S, Wu K, Yan L, Li M, Yang Z, Liu H, Zhao $\mathrm{H}$ et al (2017) A Recurrent Missense Mutation in ZP3 Causes Empty Follicle Syndrome and Female Infertility. Am J Hum Genet 101:459-465

Feng R, Sang Q, Kuang Y, Sun X, Yan Z, Zhang S, Shi J, Tian G, Luchniak A, Fukuda $Y$ et al (2016) Mutations in TUBB8 and Human Oocyte Meiotic Arrest. N Engl J Med 374:223-232

Huang HL, Lv C, Zhao YC, Li W, He XM, Li P, Sha AG, Tian X, Papasian CJ, Deng HW et al (2014) Mutant ZP1 in familial infertility. N Engl J Med 370:1220-1226

Jin F, Hamada M, Malureanu L, Jeganathan KB, Zhou W, Morbeck DE, van Deursen JM (2010) Cdc20 is critical for meiosis I and fertility of female mice. PLoS Genet 6:e1001147

Jones KT (2011) Anaphase-promoting complex control in female mouse meiosis. Results Probl Cell Differ 53:343-363

Li L, Fan L, Peng N, Yang L, Mou L, Huang W (2017) R383C mutation of human CDC20 results in idiopathic non-obstructive azoospermia. Oncotarget 8:99816-99824
Malureanu L, Jeganathan KB, Jin F, Baker DJ, van Ree JH, Gullon O, Chen Z, Henley JR, van Deursen JM (2010) Cdc20 hypomorphic mice fail to counteract de novo synthesis of cyclin B1 in mitosis. J Cell Biol 191:313-329

Musacchio A, Hardwick KG (2002) The spindle checkpoint: structural insights into dynamic signalling. Nat Rev Mol Cell Biol 3:731-741

Nasmyth K, Haering CH (2005) The structure and function of SMC and kleisin complexes. Annu Rev Biochem 74:595-648

Reis A, Madgwick S, Chang HY, Nabti I, Levasseur M, Jones KT (2007) Prometaphase APCcdh1 activity prevents non-disjunction in mammalian oocytes. Nat Cell Biol 9:1192-1198

Sang Q, Zhang Z, Shi J, Sun X, Li B, Yan Z, Xue S, Ai A, Lyu Q, Li W et al (2019) A pannexin 1 channelopathy causes human oocyte death. Sci Transl Med 11:eaav8731

Wolthuis R, Clay-Farrace L, van Zon W, Yekezare M, Koop L, Ogink J, Medema R, Pines J (2008) Cdc20 and Cks direct the spindle checkpoint-independent destruction of cyclin A. Mol Cell 30:290302

Xu Y, Shi Y, Fu J, Yu M, Feng R, Sang Q, Liang B, Chen B, Qu R, Li $B$ et al (2016) Mutations in PADI6 Cause Female Infertility Characterized by Early Embryonic Arrest. Am J Hum Genet 99:744-752
Lin Zhao, Songguo Xue, and Zhongyuan Yao have contributed equally to this work.

Electronic supplementary material The online version of this article (https://doi.org/10.1007/s13238-020-00756-0) contains supplementary material, which is available to authorized users. 